n.tronos

glyndîn

Glyndŵr University

Glyndŵr University Research Online

Computing

Computer Science

$1-1-2001$

\title{
Autofocus for inverse synthetic aperture radar (ISAR) imaging
}

Zhishun She

Glyndwr University, z.she@glyndwr.ac.uk

D A. Gray

R E. Bogner

Follow this and additional works at: http://epubs.glyndwr.ac.uk/cair

Part of the Computer and Systems Architecture Commons

\section{Recommended Citation}

She, Z., Gray, D. A., \& Bogner, R.E. (2001) 'Autofocus for inverse synthetic aperture radar (ISAR) imaging'. Signal Processing, 81(2), 275- 291

This Article is brought to you for free and open access by the Computer Science at Glyndŵr University Research Online. It has been accepted for inclusion in Computing by an authorized administrator of Glyndŵr University Research Online. For more information, please contact

d.jepson@glyndwr.ac.uk. 


\title{
Autofocus for inverse synthetic aperture radar (ISAR) imaging
}

\begin{abstract}
Autofocus is a key step of inverse synthetic aperture radar (ISAR) imaging. In this paper four new approaches to autofocussing based on the application of beamforming and subspace concepts to ISAR imaging are developed. Their relations to maximum likelihood (ML) estimation are identified. A common feature of these techniques is the estimation of the complex vector formed by the exponential function of phase rather than phase itself so that phase unwrapping is obviated. The Cramer Rao lower bound (CRLB) of the estimated complex vector corresponding to translational motion and the CRLB of the estimated distance between two scatterers are derived. The results of processing simulated and real data confirm the validity of proposed approaches.
\end{abstract}

\section{Keywords}

inverse synthetic aperture radar (ISAR); autofocus, beamforming, subspace method, array calibration

\section{Disciplines}

Computer and Systems Architecture | Computer Engineering

\section{Comments}

2001 Elsevier Science B.V. All rights reserved. This is the author's final version after peer review. This article was originally published in the Signal Processing Journal in 2001 by Elsevier. The full article can be found at http://www.sciencedirect.com 


\title{
Autofocus for inverse synthetic aperture radar (ISAR) imaging
}

\author{
Zhishun She ${ }^{1}$ D.A.Gray ${ }^{2,3}$, R.E.Bogner ${ }^{2,3}$ \\ 1 School of Informatics, University of Wales, Bangor, Gwynedd, LL57 1UT, U.K. \\ 2 Department of Electrical and Electronic Engineering, The University of \\ Adelaide, Adelaide, SA5005, Australia \\ 3 Cooperative Research Centre for Sensor Signal and Information Processing \\ (CSSIP), Adelaide, SA5095, Australia
}

\begin{abstract}
Autofocus is a key step of inverse synthetic aperture radar (ISAR) imaging. In this paper four new approaches to autofocussing based on the application of beamforming and subspace concepts to ISAR imaging are developed. Their relations to maximum likelihood (ML) estimation are identified. A common feature of these techniques is the estimation of the complex vector formed by the exponential function of phase rather than phase itself so that phase unwrapping is obviated. The Cramer-Rao lower bound (CRLB) of the estimated complex vector corresponding to translational motion and the CRLB of the estimated distance between two scatterers are derived. The results of processing simulated and real data confirm the validity of proposed approaches.
\end{abstract}

Key words: inverse synthetic aperture radar (ISAR), autofocus, beamforming, subspace method, array calibration

*Corresponding author

Ph: 441248 382722, Fax: 441248 361429, Email: zshedsees.bangor.ac.uk

\subsection{Introduction}

Synthetic aperture radar (SAR) is typically carried on a moving platform which is intended to be used in air-to-ground imaging of terrain [1]. ISAR, the inverse mode of SAR [2] [3], is usually deployed on the ground and is used for ground-toair imaging of non-cooperative moving targets such as missiles, satellites, aircraft, ships and celestial objects in the far field. ISAR has been applied widely for radar astronomy and military purposes since it can be exploited whether it is cloudy or foggy and day or night.

ISAR utilises the range-Doppler principle to achieve the desired resolution. The high range resolution is obtained by transmitting wide-band signals and the high cross-range resolution is produced by using the Doppler frequency gradient generated by the rotation of the object relative to radar-line-of-sight (RLOS). Two techniques common to SAR and ISAR signal processing are motion compensation and image formation. Motion compensation is the removal of the translational motion between object and radar prior to image formation. However, motion compensation in ISAR may be more challenging than SAR because ISAR objects are often not cooperative. 
Motion compensation of ISAR can be carried out in two steps. The first step is range realignment in which the high resolution range profiles are aligned in the range direction by placing the returns of different pulses from the same scatterer in the same range cell. It is a coarse compensation of translational motion. The second step is phase compensation, which removes the residual translational motion by multiplying the range aligned signals with the conjugate phase of a selected reference point. Phase compensation is the fine compensation of translational motion. It is usually called autofocus with the reference point being termed the focal point.

Many ISAR autofocus methods have appeared. A simple approach to ISAR autofocus is to choose as the reference point a range cell containing a strong scatterer ${ }^{[4]}$. For a complex target that does not have a stable prominent scatterer, an estimate of the pulse-to-pulse phase difference of the reference point can be made by taking the phase differences for each range cell and averaging them weighted by the amplitudes of the content of each range cell ${ }^{[5]}$. An alternative is to average the phase differences in the range cells where only strong scatterers exist[6][7][16]. However phase unwrapping is crucial for these approaches because phase averaging is needed for estimating the phase of the translational motion. Another method, based on image contrast, has also been proposed recently for ISAR autofocus [13][14]. In these approaches, many images are produced with different focusing parameters. One that produces the best image contrast is selected as the optimal focusing parameter. However the computational load of these approaches is expensive.

Phase unwrapping may be appropriate in a low-noise environment in which the amplitude of the signal never approaches zero. However in the more realistic high-noise environment, the phase unwrapping may become ambiguous [8]. In this paper, we develop some approaches for ISAR autofocussing which obviate phase unwrapping by estimating the complex exponential signal vector whose phase corresponds to the translational motion rather than the phase itself. After the complex signal vector is estimated, ISAR autofocus can be conducted by compensating all the range profiles with the complex signal vector. The block diagram of ISAR processing without phase unwrapping is shown in Fig. 1.

Fig.1 ISAR processing diagram without phase unwrapping

This paper considers ISAR autofocus as a problem of array processing and solves it from the perspective of array calibration. Four new approaches based on array processing theory for estimating the complex vector of translational motion for ISAR autofocussing are developed. The first and second approaches make use of conventional and optimum beamforming concepts. The third and fourth approaches use signal and noise subspaces of an estimated covariance matrix respectively. They have a computational advantage over the image contrast method as numerous images with different focusing parameters to calculate image contrast need not to be produced.

In section 2, a mathematical model of the observed ISAR signal is given. Section 3 describes the four proposed approaches in detail. By using the covariance matrix of the received signal rather than the phase of the received signal the problem of phase unwrapping is eliminated. Computer simulation results are presented in section 4. In section 5 the relations of the proposed approaches to the ML estimation and other ISAR autofocus methods are investigated. The CRLB of the complex vector estimation and the CRLB of the estimated distance between two scatterers are derived. In section 6 the statistical performances of the developed approaches are estimated and compared with the CRLBs. Section 7 
discusses the processing of real ISAR data.

\section{2 .0 ISAR autofocus model}

Suppose that a moving object is flying in a straight line ox as shown in Fig.2. The motion of a rigid object can be decomposed into two parts: translational motion of a certain reference point o on the object and rotational motion of the object about the point 0 . Let the Cartesian coordinates xoy be fixed on the object with range along the y-axis and cross-range along the $x$-axis when the object is at a broadside position to the radar. The radar transmits $\mathrm{M}$ steppedfrequency bursts. The aspect angle of the object relative to the RLos and the distance from the radar to the point 0 when the mth burst is sent are represented by $\theta_{m}$ and $R_{o m}$, respectively, where $m=0, \ldots, M-1$.

\section{Fig.2 ISAR imaging geometry}

Assume that there are $\mathrm{K}$ scatterers on the object. The $\mathrm{kth}$ scatterer is situated a distance $r_{k m}$ from the radar when the mth burst is sent. The range between radar and the kth scatterer with coordinate $\left(r_{k}, \phi_{k}\right)$ or $\left(x_{k}, y_{k}\right)$ is given by

$$
r_{k m}=\left[R_{o m}^{2}+r_{k}^{2}+2 R_{o m} r_{k} \sin \left(\theta_{m}+\phi_{k}\right)\right]^{1 / 2} \text {. }
$$

If the distance to the object is much larger than the size of the object, that is, $R_{o m} \gg r_{k}$, we have the approximation;

$$
r_{k m} \cong R_{o m}+x_{k} \sin \theta_{m}+y_{k} \cos \theta_{m} \text {. }
$$

Let $\rho_{\mathrm{k}}$ denote the complex reflected signal of the kth scatterer which is assumed to be independent of the illuminating frequency and the aspect angle. For each burst, L stepped frequencies $f_{1}=f_{0}+l \Delta f, I=0, \ldots, L-1$, are used where $f_{0}$ and $\Delta f$ are the initial and step frequency, respectively. The received signal $\mathrm{S}_{\mathrm{klm}}$ resulting from the kth scatterer and the lth illuminating frequency during the mth burst can be written as

$$
S_{k l m}=\rho_{k} \exp \left\{-j 4 \pi f_{l} r_{k m} / c\right\} .
$$

The total returned signal $s_{l m}$ caused by the lth illuminating frequency of the mth burst is

$s_{l m}=\sum_{k=1}^{K} \rho_{k} \exp \left\{-j 4 \pi f_{l} r_{k m} / c\right\}+w_{l m}=\sum_{k=1}^{K} \rho_{k} \exp \left\{-j 4 \pi\left(f_{0}+l \Delta f\right) r_{k m} / c\right\}+w_{l m}$.

After the pulse compression in the range direction using an inverse discrete Fourier transform (IDFT) [3] and substitution of (2) and (4), the complex envelope in the nth range cell of the mth burst becomes

$$
\begin{aligned}
& z_{n m}=\sum_{l=0}^{L-1} s_{l m} \exp \{j 2 \pi n l / L\}=\sum_{k=1}^{K} \rho_{k} \exp \left\{-j 4 \pi f_{0} r_{k m} / c\right\} \sum_{l=0}^{L-1} \exp \left\{j\left(\frac{2 \pi}{L} n-\frac{4 \pi}{c} \Delta f r_{k m}\right) l\right\}+w_{n m}^{\prime} \\
& =\exp \left\{-j 4 \pi f_{0} R_{o m} / c\right\} e_{n m}+w_{n m}^{\prime}
\end{aligned}
$$

where

$$
\begin{gathered}
e_{n m}=\sum_{k=1}^{K} \rho_{k} \exp \left\{-j 4 \pi f_{0}\left(x_{k} \sin \theta_{m}+y_{k} \cos \theta_{m}\right) / c\right\} \frac{\sin \left[L\left(\pi n / L-2 \pi \Delta f r_{k m} / c\right)\right]}{\sin \left[\left(\pi n / L-2 \pi \Delta f r_{k m} / c\right)\right]} \\
\exp \left\{j(L-1)\left(\pi n / L-2 \pi \Delta f r_{k m} / c\right)\right\},
\end{gathered}
$$

$W^{\prime}{ }_{n m} m=0, \ldots, M-1 \quad n=0, \ldots, N-1$ is the complex envelope of the additive noise and $N$ is the total number of range cells. We assume that $\mathrm{W}^{\prime}{ }_{\mathrm{nm}}$ is independent identically 
distributed complex Gaussian noise components with zero mean and variance $\sigma_{w}^{2}$.

(5) indicates that the ISAR received signal consists of two terms. One is $\exp \left\{-j 4 \pi f_{0} R_{o m} / c\right\}$ induced by the translational motion of object which should be compensated prior to image formation. The other is $e_{n m}$ corresponding to the rotational motion of object, is used to reconstruct ISAR image.

Following range compression, range realignment is done to align the high resolution range profiles in the range direction so that the returns of different pulses from the same scatterer lie in the same range cell. After range realignment is accomplished, it holds that $r_{k(m+1)} \cong r_{k m} \mathrm{~m}=0, \ldots, \mathrm{M}-2$. If only the translational motion for ISAR autofocus is considered and the rotational motion for image formation is ignored ${ }^{[6]}$, a good approximation is $\theta_{m+1} \cong \theta_{m} \mathrm{~m}=0, \ldots, \mathrm{M}-2$. These approximations, discussed below, allow the signal model $z_{n m}$ of ISAR autofocus to be written as

where

$$
z_{n m} \cong \exp \left\{-j 4 \pi f_{0} R_{o m} / c\right\} e_{n 0}+w_{n m}^{\prime}
$$

$$
\begin{gathered}
e_{n 0} \cong \sum_{k=1}^{K} \rho_{k} \exp \left\{-j 4 \pi f_{0}\left(x_{k} \sin \theta_{0}+y_{k} \cos \theta_{0}\right) / c\right\} \frac{\sin \left[L\left(\pi n / L-2 \pi \Delta f r_{k 0} / c\right)\right]}{\sin \left[\left(\pi n / L-2 \pi \Delta f r_{k 0} / c\right)\right]} \\
\exp \left\{j(L-1)\left(\pi n / L-2 \pi \Delta f r_{k 0} / c\right)\right\} .
\end{gathered}
$$

The complex envelope vector in the nth range cell can be expressed as the desired signal model

$$
Z_{n}=e_{n 0} D+W_{n}
$$

where $Z_{n}=\left[z_{n o}, \ldots, z_{n(M-1)}\right]^{T}, \quad W_{n}=\left[w_{n 0}^{\prime}, \ldots, w_{n(M-1)}^{\prime}\right]^{T}, \lambda$ is the wavelength corresponding to $f_{0}$, and $D=\left[\exp \left\{-j 4 \pi R_{o 0} / \lambda\right\}, \ldots, \exp \left\{-j 4 \pi R_{o(M-1)} / \lambda\right\}\right]^{T}$ which is the complex vector that ISAR autofocus needs to estimate. (9) is the vector form of signal model for ISAR autofocus.

Although the above signal model is derived by use of a stepped frequency waveform, it is straightforward to generalize it to other signal waveforms such as the short pulse and chirp pulse-compression waveforms. The above derivation shows that the signal model (9) is valid after both the range compression and the range realignment have been accomplished for ISAR autofocus. Finally it is worth noticing that the complex vector $D$ is space invariant and does not correspond to a particular strong scatterer.

In order to satisfy the approximation $\theta_{m+1} \cong \theta_{m}$, the phase variation induced by the rotational motion should be less than $\pi / 2$ corresponding to a range error of $\lambda / 8^{[1]}$. Consider two adjacent $\mathrm{mth}$ and $(\mathrm{m}+1)$ th pulses; the exact signal returns from one scatterer are

$$
\begin{gathered}
e_{n m}=\rho_{1} \exp \left\{-j 4 \pi\left(x_{1} \sin \theta_{m}+y_{1} \cos \theta_{m}\right) / \lambda\right\} \frac{\sin \left[L\left(\pi n / L-2 \pi \Delta f r_{1 m} / c\right)\right]}{\sin \left[\left(\pi n / L-2 \pi \Delta f r_{1 m} / c\right)\right]} \\
\quad \exp \left\{j(L-1)\left(\pi n / L-2 \pi \Delta f r_{1 m} / c\right)\right\} . \\
e_{n(m+1)}=\rho_{1} \exp \left\{-j 4 \pi\left(x_{1} \sin \theta_{m+1}+y_{1} \cos \theta_{m+1}\right) / \lambda\right\} \frac{\sin \left[L\left(\pi n / L-2 \pi \Delta f r_{1(m+1)} / c\right)\right]}{\sin \left[\left(\pi n / L-2 \pi \Delta f r_{1(m+1)} / c\right)\right]} \\
\exp \left\{j(L-1)\left(\pi n / L-2 \pi \Delta f r_{1(m+1)} / c\right)\right\} .
\end{gathered}
$$

and the phase variation due to the rotational motion is 


$$
\Delta \phi_{m}=4 \pi\left[x_{1}\left(\sin \theta_{m+1}-\sin \theta_{m}\right)+y_{1}\left(\cos \theta_{m+1}-\cos \theta_{m}\right)\right] / \lambda .
$$

Limiting $\Delta \phi_{\mathrm{m}}$ to be less than $\pi / 2$, we have

$$
\left|x_{1}\left(\sin \theta_{m+1}-\sin \theta_{m}\right)\right|+\left|y_{1}\left(\cos \theta_{m+1}-\cos \theta_{m}\right)\right|<\lambda / 8 \text {. }
$$

By use of $\sin \left(\delta \theta_{m} / 2\right) \cong \delta \theta_{m} / 2$ for small $\delta \theta_{\mathrm{m}} / 2$ where $\delta \theta_{m}=\theta_{m+1}-\theta_{m}$, we have

$$
\delta \theta_{m}<\frac{\lambda}{8\left\{\left|x_{1} \cos \left[\left(\theta_{m+1}+\theta_{m}\right) / 2\right]+\right| y_{1} \sin \left[\left(\theta_{m+1}+\theta_{m}\right) / 2\right]\right\}} .
$$

Thus the sample interval $l_{s}$ of the synthetic aperture must satisfy

$$
l_{s}=R_{o} \delta \theta_{m}<\frac{\lambda R_{o}}{8\left\{\left|x_{1} \cos \left[\left(\theta_{m+1}+\theta_{m}\right) / 2\right]+\right| y_{1} \sin \left[\left(\theta_{m+1}+\theta_{m}\right) / 2\right]\right\}}
$$

where $R_{0}$ is the distance between the radar and object. Fig.3 shows the required sampling interval versus the aspect angle where the parameters are chosen as $\lambda=3 \mathrm{~cm}, \mathrm{x}_{1}=1 \mathrm{~m}$ and $\mathrm{y}_{1}=1 \mathrm{~m}$. It indicates that the required sampling interval decreases as the aspect angle increases. The minimum sampling intervals are $26.51 \mathrm{~m}$ and $79.53 \mathrm{~m}$ when the $R_{\circ}$ equals to $10 \mathrm{~km}$ and $30 \mathrm{~km}$, respectively. This condition can be satisfied by increasing the PRF.

Fig.3 Sampling interval versus aspect angle

One requirement for range realignment to satisfy the approximation $r_{k(m+1)} \cong r_{k m}$ is that the variation of relative position between scatterers in range profile should be less than one range resolution cell. Consider an example of two scatterers as shown in Fig.4, they are at first located at A and B. During the interval of two adjacent pulses, they rotate to positions $\mathrm{A}_{1}$ and $\mathrm{B}_{1}$. The initial and final projections of two scatterers on range direction (y axis) are dcos $\theta_{m}$ and $d \cos \theta_{m+1}$, respectively where $d$ is the distance between two scatterers. The variation of relative position between two scatterers in range profiles needs to satisfy $d \mid \cos \theta_{m+1}-\cos \theta_{m} k \tau_{r}$ where $\tau_{r}$ is the range resolution. By use of $\sin \left(\delta \theta_{m}\right) \cong \delta \theta_{m}$, this relation becomes

$$
\delta \theta_{m}<\frac{\tau_{r}}{d \sin \left[\left(\theta_{m+1}+\theta_{m}\right) / 2\right]} .
$$

This is a weak requirement as compared with (14) if $\lambda<\tau_{r}$.

Fig.4 Position variation of two scatterers

\subsection{Four autofocus approaches}

In standard ISAR autofocussing algorithms, $R_{o m}$ is estimated and the range aligned signals are corrected with the phase term $\exp \left\{-j 4 \pi R_{o m} / \lambda\right\}$. However, from (7) it can be seen that for the above signal model, we only need to work with the $z_{n m}^{\prime}=z_{n m} \exp \left\{j 4 \pi R_{o m} / \lambda\right\}$. Thus it is unnecessary to estimate $R_{o m}$ and all that is required is an estimate of $\exp \left\{j 4 \pi R_{o m} / \lambda\right\}$, obviating the need for phase unwrapping. In this section, we will develop the four new approaches for estimating the $\exp \left\{-j 4 \pi \mathrm{R}_{\mathrm{om}} / \lambda\right\} \mathrm{m}=0, \ldots, \mathrm{M}-1$, that is the complex vector $D$ rather than $R_{o m}$.

\subsection{Conventional beamforming approach}

The first approach is an extension of the conventional beamforming. It is assumed that the conventional beamformer with uniform shading across the array sets the weights to be equal to the steering vector ${ }^{[10]}$. In conventional 
beamforming the steering vector is parameterized by the steering angle; however for the present application it is parameterized by the $R_{o m}$, namely, the range between the radar and the object when the mth burst is transmitted. Loosely, this may be thought of as beamforming in range. Thus the beamforming weight vector $v$ is defined as

$$
v=\left[v_{0}, \ldots, v_{(M-1)}\right]^{T}
$$

where $v_{m}=\exp \left\{-j 4 \pi R_{o m} / \lambda\right\} \mathrm{m}=0, \ldots, \mathrm{M}-1$. The output of the conventional beamforming is

$$
P_{c}=v^{H} C_{n} v / M^{2}
$$

where $C_{n}$ is the covariance matrix of the signal vector in the nth range cell which is defined as

$$
C_{n}=E\left\{Z_{n} Z_{n}^{H}\right\}
$$

where $\mathrm{H}$ denotes the Hermitian transpose and $\mathrm{E}$ denotes ensemble averaging. Based on (9), (19) reduces to

$$
C_{n}=p_{n} D D^{H}+\sigma_{w}^{2} I_{M}
$$

where $p_{n}=\left|e_{n 0}\right|^{2}$ is the signal power of the nth range cell and $I_{M}$ is the identity matrix.

The first approach for estimating $D$ can be defined as the maximization of the conventional beamforming output by choice of $\mathrm{v}$, that is,

$$
\max \left\{v^{H} C_{n} v / M^{2}\right\} \quad \forall v
$$

subject to the constraint $v^{H} v=M$. The above optimization happens when the estimated steering vector $v$ is equal to the eigenvector $u_{1}$ of $\lambda_{1}$, that is,

$$
\hat{v}=u_{1}=D / M
$$

where $\lambda_{1}$ is the maximal eigenvalue of $C_{n}$. Then (22) means that $\mathrm{P}_{\mathrm{c}}$ attains its maximal value when the estimated translational motion phase matches the actual translational motion phase.

\subsection{Optimum beamforming approach}

The second new approach is based on optimum beamforming. With the conventional beamforming, the weights are calculated using the inverse of the covariance matrix for the noise alone. In practice, the estimated covariance matrix contains the signals as well as noise, which leads to the beamformer treating the signal as noise and suppressing it. To overcome this problem, the optimum beamformer calculates the weights so that the output of the beamformer is minimized while the response in the direction of the desired signal is constrained to unity. The output of the optimum beamformer is ${ }^{[10]}$

$$
P_{o}=\left(v^{H} C_{n}^{-1} v\right)^{-1} \text {. }
$$

The second approach to estimate $D$ is obtained by the maximization of the optimum beamforming output, that is,

$$
\max \left\{\left(v^{H} C_{n}^{-1} v\right)^{-1}\right\} \quad \forall v
$$

with constraint $v^{H} v=M$. This maximization occurs when the estimated steering vector $\mathrm{v}$ is equal to the eigenvector $\mathrm{u}_{1}$ of $\mathrm{C}_{\mathrm{n}}$ corresponding to the maximal eigenvalue, that is,

$$
\hat{v}=u_{1}=D / M \text {. }
$$


(25) also shows that $\mathrm{P}_{\circ}$ reaches its maximal value when the estimated translational motion phase approaches the actual translational motion phase.

When the covariance matrix becomes singular, the inverse of covariance matrix $C_{n}^{-1}$ may be replaced by the Moore-Penrose pseudoinverse of covariance matrix $C_{n}^{+}$.

\subsection{Signal subspace approach}

The third approach is to exploit the eigendecomposition of covariance matrix. This technique has been applied to shape estimation of sonar towed array ${ }^{[1]}$ and correction of SAR phase error ${ }^{[9]}$. Here it is extended to ISAR autofocus. Based on (20), it has been shown that the dimension of signal subspace of $C_{n}$ is $1^{[12]}$. The eigenvalue and eigenvector corresponding to signal subspace are

$$
\lambda_{1}=p_{n} M+\sigma_{w}^{2}
$$

and

$$
u_{1}=D / M \text {. }
$$

The matrix that projects data onto signal subspace is

$$
P_{s}=D D^{H} / M
$$

which has eigenvalues of 1 and 0 . The signal subspace approach maximizes the projection of the steering vector onto the signal subspace, that is,

$$
\max \left\{\left\|P_{s} v\right\|^{2}\right\} \quad \forall v
$$

with constraint $v^{H} v=M$ where || $\mid$ is the Euclidean norm of a vector. With the relation of $P_{s}^{2}=P_{s}$, (29) is equivalent to

$$
\max \left\{v^{H} P_{s} v\right\}
$$

subject to $v^{H} v=M$. This optimization happens when the steering vector is equal to the eigenvector corresponding to the maximal eigenvalue of $\mathrm{P}_{s}$, that is,

$$
\hat{v}=u_{1}=D / M \text {. }
$$

\subsection{Noise subspace approach}

Based on (20), the dimension of the noise subspace is $M-1$. All the eigenvalues of the noise subspace are equal to $\sigma_{w}^{2}$ and their corresponding eigenvectors are denoted by $u_{2}, \ldots, u_{M}$. The projection matrix onto the noise subspace is

$$
P_{n}=\left(u_{2}, \ldots, u_{M}\right)\left(u_{2}, \ldots, u_{M}\right)^{H} \text {. }
$$

Noise subspace approach is to minimize the projection of steering vector onto the noise subspace, namely,

$$
\min \left\{\left\|P_{n} v\right\|^{2}\right\} \quad \forall v
$$

with constraint $v^{H} v=M$ which is equivalent to

$$
\min \left\{v^{H} P_{n} v\right\}
$$

subject to $v^{H} v=M$. With the relation of $P_{n}=I-D D^{H} / M$, the above minimum occurs if

$$
\hat{v}=D / M \text {. }
$$




\subsection{Computer simulation}

Computer simulations were conducted to verify the validity of the developed approaches for ISAR autofocus. In the simulation, the scattering model of an aircraft is shown in Fig.5. The return from the aircraft was assumed to be dominated by scattering from its nose, engine intakes and exhausts, wing pods and horizontal stabiliser extremities with meter coordinates $(11,0),(0,2)$, $(0,-2),(-3.3,2),(-3.3,-2),(0,8),(0,-8),(-9,3)$, and $(-9,-3)$. The reflectivity ratios of the nine scatterers were supposed to be $1: 3: 3: 2: 2: 1: 1: 1: 1$. The radar transmitted a stepped-frequency waveform with an effective bandwidth of $50 \mathrm{MHz}$ which results in a $3 \mathrm{~m}$ resolution in range. The number of stepped frequencies was 16 . The radar wavelength was $3 \mathrm{~cm}$. The initial distance between the radar and the aircraft was $30 \mathrm{~km}$ and initial aspect angle was $0^{\circ}$. The aircraft was flying in a straight line with a speed of $200 \mathrm{~m} / \mathrm{s}$. The total change of angle was $0.38^{\circ}$ which provided a $2 \mathrm{~m}$ resolution in cross-range.

\section{Fig.5 Scattering model of simulated aircraft}

The received signals were generated with noise added to give SNR=20dB which were processed by range compression, range realignment, autofocus, and range-Doppler imaging. The covariance matrix was estimated by averaging over all the range cells. Fig. 6 shows the ISAR images of simulated aircraft: (a) the ISAR image without autofocus, (b) the ISAR image focused by the conventional beamformer approach, (c) the ISAR image focused by the optimum beamformer approach, (d) the ISAR image focused by the signal subspace approach and (e) the ISAR image focused by the noise subspace approach. The reconstructed ISAR images have correspondences to the scattering model of simulated aircraft as shown in Fig.5. The small difference among (b), (c) and (d) can be attributed to imperfection in the optimization associated with the size of the search steps.

\section{(a) Unfocused image}

(b) Focused image with the conventional beamforming approach

(c) Focused image with the optimum beamforming approach

(d) Focused image with the signal subspace approach

(e) Focused image with the noise subspace approach Fig. 6 ISAR images of simulated aircraft

\subsection{Relations to maximum likelihood estimation and other methods and the Cramer- Rao lower bounds}

The derivations in section 3.0 are based on the assumption that the exact covariance matrices are available. However in practice these must be estimated from a finite number of data samples. In this section we consider the problem from an estimation perspective.

By inspection of (9) the vector $D$ is independent of range cell index and so the covariance matrix can be estimated by replacing the ensemble average with one over range cells, namely,

$$
\hat{C}=\frac{1}{N} \sum_{n=0}^{N-1} Z_{n} Z_{n}^{H} \text {. }
$$


This ensures that it will have sufficient rank for the techniques to work and reduce the effect of rotational motion.

\subsection{Relation to maximum likelihood estimation}

If the received noise is Gaussian distributed, the joint probability density function of $Z=\left[Z_{0}, \ldots, Z_{N-1}\right]$ is given by

$$
p(Z ; \Theta)=\pi^{-M N}\left|C_{n}\right|^{-N} \exp \left\{-N \operatorname{Tr}\left(C_{n}^{-1} \hat{C}\right)\right\}
$$

where the unknown parameters $\Theta=\left\{D, p_{n}\right\}$. Maximizing the log probability density function with respect to $\Theta$ results in the following equation [9] [11]

$$
\hat{C} \hat{u}_{1}=\sigma_{w}^{2} \lambda_{1} \hat{u}_{1}
$$

(38) shows that the eigenvector $\hat{u}_{1}$ of $\hat{C}$ corresponding to the maximal eigenvalue is the ML estimation of the complex signal vector $D$.

\subsection{Relation to other autofocus methods}

If a dominant strong scatterer exists in the kth range cell, $\hat{C}$ can be approximated as

$$
\hat{C} \approx Z_{k} Z_{k}^{H}+\hat{\sigma}_{1}^{2} I \text {. }
$$

In this case, the largest eigenvalue of $\hat{C}$ is $\left\|Z_{k}\right\|^{2}+\hat{\sigma}_{1}^{2}$ and the corresponding eigenvector is

$$
\hat{u}_{1}=Z_{k}
$$

which is equivalent to the strong scatterer referencing method ${ }^{[4]}$.

Subaperture processing ${ }^{[16]}$ can be applied to the developed approaches to reduce the computational load. Consider a special case where each subaperture consists of two pulses and all the subapertures are connected with one pulse. For the mth subaperture, the covariance matrix in the nth range cell is expressed as

$$
C_{n}=p_{n}\left[\begin{array}{cc}
1 & \exp \left\{j 4 \pi\left(R_{o(m+1)}-R_{o m}\right) / \lambda\right\} \\
\exp \left\{-j 4 \pi\left(R_{o(m+1)}-R_{o m}\right) / \lambda\right\} & 1
\end{array}\right]
$$

and the eigenvector corresponding to the maximum eigenvalue is

$$
u_{1}=\left[\exp \left\{-j 4 \pi\left(R_{o(m+1)}^{1}-R_{o m}\right) / \lambda\right\}\right] \text {. }
$$

However in practice we have to estimate the covariance matrix by range cell averaging, that is,

$$
\hat{C}=\frac{1}{N} \sum_{n=1}^{N}\left[\begin{array}{c}
z_{n m} \\
z_{n(m+1)}
\end{array}\right]\left[\begin{array}{ll}
z_{n m}^{*} & z_{n(m+1)}^{*}
\end{array}\right] .
$$

The eigenvector corresponding to largest eigenvalue of $\hat{C}$ is

$$
\hat{u}_{1}=\left[p_{1} \sum_{n=1}^{N} z_{n m}^{*} z_{n(m+1)}\right]
$$

where $p_{1}$ is a scale factor. Thus we have an estimation

$$
\exp \left\{-j 4 \pi\left(\hat{R}_{o(m+1)}-\hat{R}_{o m}\right) / \lambda\right\}=\left(\sum_{n=1}^{N} z_{n m}^{*} z_{n(m+1)}\right) /\left|\sum_{n=1}^{N} z_{n m}^{*} z_{n(m+1)}\right|
$$


which is equivalent to Doppler centroid tracking ${ }^{[5]}$.

\subsection{CRLB of the estimated complex signal vector $D$}

The Cramer-Rao lower bound (CRLB) gives the minimum variance that an unbiased estimator can achieve and is a useful tool for quantifying the performance of practical estimators. In (9), $D$ is the complex vector associated with the translational motion of the object. The variance of estimated $D$ compared with the CRLB of $D$ indicates the efficiency of ISAR autofocus. The CRLB of $D$ is $g i v e n$ below, summarized from [15].

In the signal model of (9), both $e_{n o}$ and $D$ are unknown. An ambiguity occurs when $e_{n o}$ and $D$ become $\alpha e_{n o}$ and $\alpha^{-1} D$, respectively. If we set constraints $e_{0}^{H} e_{0}=N$ where $e_{0}=\left(e_{00}, \ldots, e_{N-1,0}\right)^{T}$ and $\operatorname{Im}\left(e_{00}\right)=0$, this ambiguity will be removed.

Let $D=u+j w$ and $e_{0}=r+j t$ with constraints $t_{0}=0$ and $e_{0}^{H} e_{0}=N$. Then the CRLBs of $u$ and $w$ are given by

$$
\begin{aligned}
& C R L B\left(u_{m}\right)=\frac{1}{2 N}(P)_{m m}+\beta w_{m}^{2} \\
& C R L B\left(w_{m}\right)=\frac{1}{2 N}(P)_{m m}+\beta u_{m}^{2}
\end{aligned}
$$

where the noise covariance matrix $C_{w}=P+j Q, u_{m}$ is the mth element of the vector $u, \beta=\left(N-p_{0}\right) /\left(2 N p_{0}\left[D^{H} C_{w}^{-1} D\right]\right)$ and $(P)_{m m}$ is the mth diagonal element of the matrix $P$. Thus the CRLB of $D_{m}$ (the mth element of $D$ ) takes the form

$$
\operatorname{CRLB}\left(D_{m}\right)=\frac{1}{N}(P)_{m m}+\beta \text {. }
$$

\subsection{CRLB of the estimated distance between two scatterers}

The variance of the estimated distance between two scatterers spaced in crossrange is a measure of ISAR location capability and the CRLB for this variance is derived below.

In this analysis, the object is composed of two scatterers $s_{1}$ and $s_{2}$ with the coordinates $\left(\mathrm{x}_{1}, \mathrm{y}_{1}\right)$ and $\left(\mathrm{x}_{2}, \mathrm{y}_{2}\right)$. When the distance between the radar and the object is much larger than the size of object, the returned signal of the mth pulse can be expressed as

$$
\begin{aligned}
z_{m}=\rho_{1} & \exp \left\{-j 4 \pi\left[r_{m}+x_{1} \sin \theta_{m}+y_{1} \cos \theta_{m}\right] / \lambda\right\} \\
& +\rho_{2} \exp \left\{-j 4 \pi\left[r_{m}+x_{1} \sin \theta_{m}+y_{1} \cos \theta_{m}\right] / \lambda\right\}+w_{m}
\end{aligned}
$$

where $\rho_{1}$ and $\rho_{2}$ are proportional to the reflectivities of $s_{1}$ and $s_{2}$, respectively, $r_{m}$ denotes the distance between the radar and the centre of the two scatterers when the $\mathrm{mth}$ pulse is sent and $\mathrm{w}_{\mathrm{m}}$ is the additive noise, assumed to be independent and identically distributed from pulse to pulse. It is modelled as complex Gaussian with zero mean and variance $\sigma_{w}^{2}$.

Assuming $\rho_{1}=\rho_{2}=\rho$ for simplicity, (49) is changed into

$$
z_{m}=2 \rho \cos \left\{2 \pi d \sin \left[\theta_{m}+\phi\right] / \lambda\right\} u_{m}+w_{m}
$$

where $\mathrm{d}$ is the distance between the two scatterers, $\phi$ is the angle between the $\mathrm{x}$ axis and a straight line through $s_{1}$ and $s_{2}$, and

$u_{m}=\exp \left\{-j 2 \pi\left[2 r_{m}+\left(x_{1}+x_{2}\right) \sin \theta_{m}+\left(y_{1}+y_{2}\right) \cos \theta_{m}\right] / \lambda\right\}$. 
If $\phi, \theta_{m}$ and $u_{m}$ are assumed to be known, the joint probability density function of single observation is

$$
p\left(z_{m} ; d\right)=\frac{1}{\pi \sigma_{w}^{2}} \exp \left\{-\frac{\left|z_{m}-2 \rho \cos \left\{2 \pi d \sin \left[\theta_{m}+\phi\right] / \lambda\right\} u_{m}\right|^{2}}{\sigma_{w}^{2}}\right\}
$$

and the joint probability density function of multiple independent observations is

$$
p\left(z_{0}, \ldots, z_{M-1} ; d\right)=\prod_{m=0}^{M-1} p\left(z_{m} ; d\right)=\left(\pi \sigma_{w}^{2}\right)^{-M} \exp \left\{-\frac{1}{\sigma_{w}^{2}} \sum_{m=0}^{M-1}\left|z_{m}-2 \rho \cos \left\{2 \pi d \sin \left[\theta_{m}+\phi\right] / \lambda\right\} u_{m}\right|^{2}\right\}
$$

Thus we get the log joint probability density function

$L\left(z_{0}, \ldots, z_{M-1} ; d\right)=-M \ln \left(\pi \sigma_{w}^{2}\right)-\frac{1}{\sigma_{w}^{2}} \sum_{m=0}^{M-1}\left|z_{m}-2 \rho \cos \left\{2 \pi d \sin \left[\theta_{m}+\phi\right] / \lambda\right\} u_{m}\right|^{2}$.

From Appendix A the CRLB of $d$ is given by

$$
C R L B(d)=\frac{\lambda^{2}}{32 \pi^{2} S N R \sum_{m=0}^{M-1} \sin \left[\theta_{m}+\phi\right]^{2} \sin \left\{2 \pi d \sin \left[\theta_{m}+\phi\right] / \lambda\right\}^{2}}
$$

where $S N R=\rho^{2} / \sigma_{W}^{2}$ denotes the signal-to-noise ratio (SNR).

It is noted that in the above derivations we have actually formed the CRLB under the assumption that $\phi,\left\{\theta_{\mathrm{m}}\right\}$ and SNR are all known. In practice this may not be the case; however for comparison of different methods this approach is justified.

\subsection{Statistical analysis}

Monte-Carlo simulations were conducted to analyse the accuracies of the four ISAR autofocus approaches. In the simulation, $\mathrm{w}_{\mathrm{nm}}$ was independent identically distributed complex Gaussian noise components with zero mean and variance $\sigma_{w}^{2}$. Thus we had $C_{w}=\sigma_{w}^{2} I_{M}$. The CRLB of the complex signal vector $D$ corresponding to the translational motion takes the form

$$
\operatorname{CRLB}\left(D_{m}\right)=\left(1-\frac{1}{2 M}\right) \frac{\sigma_{w}^{2}}{N}+\frac{1}{2 M S N R}
$$

where $S N R=p_{0} / \sigma_{w}^{2}$. It is noted that $e_{0}^{H} e_{0}$ is the total power of the received signals over the $N$ range cells. Ignoring the variability from range to range of the signal power, we may approximate $e_{0}^{H} e_{0}$ as $N p_{0}$. Furthermore in the derivation of the CRLB it is assumed that $e_{0}^{H} e_{0}=N$. Thus we may replace the term $\sigma_{w}^{2} / N$ in the above equation by $\sigma_{w}^{2} / N p_{0}$ or $1 /(N S N R)$.

The parameters of ISAR simulation were described in section 4. The statistics were based on 100 simulations for each estimator at the specified SNR level. The mean square errors of the complex signal vector estimated by the conventional beamforming approach $(\mathrm{PC})$, the optimum beamforming approach (Po), the signal subspace approach (Ps) and the noise subspace approach (Pn) are shown in Fig.7 and compared with the CRLB of the complex signal vector for $\mathrm{M}=128$ and $\mathrm{N}=10$. It indicates that PC, Ps and Pn approach the CRLB when $S N R \geq 0 d B$ and Po reaches the CRLB as $S N R \geq 5 d B$. This is because that Po uses the inverse of covariance matrix which makes it more sensitive to noise than other estimators. Fig.7 indicates that the SNR thresholds (minimum required SNRs) for ISAR autofocus by use of $\mathrm{PC}, \mathrm{PO}, \mathrm{Ps}$ and $\mathrm{Pn}$ are $0 \mathrm{~dB}, 5 \mathrm{~dB}, 0 \mathrm{~dB}$ and $0 \mathrm{~dB}$, respectively. At high SNR above the SNR thresholds the mean square errors of the four methods approach to 
the CRLB. However the CRLB fails to predict the mean square errors when SNR drops below the SNR thresholds. For low SNR the theoretical analysis of statistical performance of estimators may be conducted by use of Weiss-Weinstein lower bound ${ }^{[17]}$.

Fig.7 Mean square error of the estimated complex signal vector

Fig. 8 gives the CRLBs for various values of $\mathrm{N}$ (the number of range cells) with $\mathrm{M}=128$ (the number of pulses) and $\sigma_{w}^{2}=1$. It shows that the CRLBs of $D$ decrease with the increase of $\mathrm{N}$ and SNR. They are approximately proportional to $1 / \mathrm{N}$ if the SNR is high enough. The CRLB reaches $0 \mathrm{~dB},-10 \mathrm{~dB}$ and $-20 \mathrm{~dB}$ when $\mathrm{N}$ is equal to 1,10 and 100, respectively, in the case of high SNR.

\section{Fig. 8 The CRLBs versus SNR for three $N$ values}

The CRLBs for $\mathrm{N}=10$ and three values of $\mathrm{M}$ are shown in Fig.9. We see that the difference between them becomes indiscernible in the case of high SNR. In other words the CRLB is almost independent of $M$ if SNR is high enough where the CRLB approaches $-10 \mathrm{~dB}$. This means that the subaperture processing is more effective for high SNR.

Fig.9 The CRLBs versus SNR for three M values

In the simulation, two scatterers were assumed to be $15 \mathrm{~m}$ apart in cross-range. After ISAR autofocus ISAR images were produced and the distance between the two scatterers was estimated by detecting the peak positions corresponding to the two scatterers. Fig.10 (a) shows the bias (mean error) of the estimated distance between two scatterers determined by PC, Po, Ps and Pn versus SNR. It indicates that the SNR thresholds of these four approaches are $-5 \mathrm{~dB}$. When SNR is higher than $-5 \mathrm{~dB}$, the bias of the four methods approaches zero. If SNR is below $-5 \mathrm{~dB}$, the bias increases.

Mean square errors of the estimated distance between two scatterers determined by the four approaches are given in Fig.10 (b) and compared with the CRLB. It is illustrated that the experimental variances of the conventional beamforming, the signal subspace and the noise subspace approaches reach the CRLB when

$S N R \geq 15 d B$. This means that they are statistically efficient. Although the optimum beamforming approach does not reach the CRLB, the mean square error is close to the CRLB as SNR increases. Fig.10 (b) shows that the minimum SNR required for precisely locating multiple scatterers is $15 \mathrm{~dB}$.

\section{(a) Bias}

(b) Mean square error

Fig.10 Bias and mean square error of the estimated distance between two scatterers

In the above simulations, it is assumed that $d, \phi,\left\{\theta_{m}\right\}$ and SNR are known precisely in order to calculate the CRLBs. For practical application this may not be true. Nevertheless it gives an effective approach to compare the performances of different methods theoretically.

\section{0 Real data results}

Thanks to Prof. B.D.Steinberg of the University of Pennsylvania, we received experimental data of a Boeing-727. The commercial Boeing-727 aircraft was flying into the Philadelphia International Airport. The range, speed and altitude of 
the aircraft were about $2.7 \mathrm{~km}, 120 \mathrm{~m} / \mathrm{s}$ and a few thousand feet, respectively. The central frequency of radar was $9.6 \mathrm{GHz}$ (X-band) $(\lambda=3.123 \mathrm{~cm})$. Range resolution of $1 \mathrm{~m}$ was achieved by transmitting a narrow pulse with the width of $7 \mathrm{~ns}$. Signals in 120 range cells were recorded and $\mathrm{PRF}$ was $400 \mathrm{~Hz}$. The real data were processed with range realignment, autofocus, and range-Doppler imaging. The ISAR images of the Boeing-727 are shown in Fig.11 where (a) is the unfocused image, (b) is the focused image with the conventional beamforming approach, (c) is the focused image with the optimum beamforming approach, (d) is the focused image with the signal subspace approach and (e) is the focused image with the noise subspace approach. Compared with the plan view of Boeing-727 as shown in Fig.9 (f), the focused images are quite impressive.

(a) Unfocused image

(b) Focused image with the conventional beamforming approach

(c) Focused image with the optimum beamforming approach

(d) Focused image with the signal subspace approach

(e) Focused image with the noise subspace approach

(f) Plan view

Fig. 11 ISAR images of Boeing-727

\section{8 . 0 Conclusions}

Four new approaches for ISAR autofocus based on the complex signal vector estimation were developed. They are the conventional beamforming, the optimum beamforming, the signal subspace and the noise subspace methods. Their advantages over conventional methods are that the returned signals are processed with the covariance matrix rather than the phase itself so that no phase unwrapping is needed. The signal subspace approach is identified as the ML estimation of the complex signal vector associated with ISAR autofocus. If a dominant strong scatterer is detected, it simplifies into the strong scatterer reference method. When the subaperture processing is used, it is related to the Doppler centroid tracking. The mean square errors compared with CRLBs show that the developed approaches are statistically efficient. The results with simulated and real data for two dimensional ISAR imaging show that the four developed approaches can provide the high quality ISAR images. The developed approaches may have far wider application to other focusing problems.

\subsection{References}

[1] D.A.Ausherman, A.Kozma, J.L.Walker, H.M.Jones and E.C.Poggio, "Developments in radar imaging," IEEE Transactions on Aerospace and Electronic Systems, Vol.20, No.4, 1984, pp.363-398.

[2] C.C.Chen and H.C.Andrews, "Target-motion-induced radar imaging," IEEE Transactions on Aerospace and Electronic Systems, Vol.16, No.1, 1980, pp.2-14. [3] D. R.Wehner, High resolution radar, Artech House, 1995.

[4] B.D.Steinberg, "Microwave imaging of aircraft," Proceedings of the IEEE, Vol.76, No.12, 1988, pp.1578-1592.

[5] M.J.Prickett and C.C.Chen, "Principles of inverse synthetic aperture radar imaging," Proceedings of EASCON, Arlington, U.S.A., 1980, pp.340-345.

[6] B.Kang, H.Subbaram and B.D.Steinberg, "Improved adaptive-beamforming target for self-calibrating a distorted phased array," IEEE Transactions on Antenna and Propagation. Vol.38, No.2, 1990, pp.186-194. 
[7] H.Wu, D.Grenier, G.Y.Delisle and D.G.Fang, "Translational motion compensation in ISAR image processing," IEEE Transactions on Image Processing, Vol.14, No.11, 1995, pp.1561-1571.

[8] R.M.Goldstein, H.A.Zebker and C.L.Werner, "Satellite radar interferometry: Two-dimensional phase unwrapping," Radio science, Vol.23, No.4, 1988, pp.713720 .

[9] C.V.Jakowatz and D.E.Wahl, "Eigenvector method for maximum-likelihood estimation of phase errors in synthetic-aperture-radar imagery," Journal of the Optical Society of Amercia, A, Vol.10, No.12, 1993, pp.2539-2546.

[10] H.A. d'Assumpcao and G.E.Mountford, "An overview of signal processing for arrays of receivers", Journal of Electrical and Electronic Engineering, Australia, Vol.4, No.1, 1984, pp.6-19.

[11] D.A.Gray, W.O.Wolfe, and J.L.Riley, "An eigenvector method for estimating the positions of the elements of an array of receivers," Proceedings of the Australian Symposium on Signal Processing Application, Adelaide, Australia, 1989, pp.391-393.

[12] R.O.Schmidt, "Multiple emitter location and signal parameter estimation", IEEE Transactions on Antenna and Propagation, Vol.34, No.3, 1986, pp.276-280. [13] Z.S.She and Z.D.Zhu, "Two improved methods of motion compensation for 1D cross-range ISAR imaging," Proceedings of IEEE NAECON, Dayton, U.S.A., May $1996, \mathrm{pp} .227-230$.

[14] F.Berizzi and G.Corsini, "Autofocusing of inverse synthetic aperture radar images using contrast optimization," IEEE Transactions on Aerospace and Electronic Systems, Vol.32, No.3, 1996, pp.1185-1191.

[15] D.A.Gray, B.G.Quinn and J.L.Riley, "Maximum likelihood estimation of array shape and the Cramer-Rao lower bound," private communication, January 1998.

[16] E.Yadin, "SAR autofocusing viewed as adaptive beamforming on prominent scatterers", Proceedings of National Radar Conference, U.S.A., 1994, pp.138-143. [17] S.Haykin et al., "Some aspects of array signal processing", IEE Proceedings, Pt.F, Vol.139, No.1, 1992, pp.1-26.

\subsection{Appendix A}

The first partial derivative of $L\left(z_{0}, \ldots, z_{M-1} ; d\right)$ with respect to $d$ is $\frac{\partial L}{\partial d}=-\frac{1}{\sigma_{w}^{2}} \sum_{m=0}^{M-1} \frac{4 \pi \rho}{\lambda} \sin \left[\theta_{m}+\phi\right] \sin \left\{\frac{2 \pi d}{\lambda} \sin \left[\theta_{m}+\phi\right]\right\}\left[u_{m} z_{m}^{*}+u_{m}^{*} z_{m}\right]+\frac{8 \pi \rho^{2}}{\lambda} \sin \left[\theta_{m}+\phi\right] \sin \left\{\frac{4 \pi d}{\lambda} \sin \left[\theta_{m}+\phi\right]\right\}$.

The second partial derivative of $L\left(z_{0}, \ldots, z_{M-1} ; d\right)$ with respect to $d$ has the form $\frac{\partial^{2} L}{\partial d^{2}}=-\frac{1}{\sigma_{w}^{2}} \sum_{m=0}^{M-1} \frac{8 \pi^{2} \rho}{\lambda} \sin \left[\theta_{m}+\phi\right]^{2} \cos \left\{\frac{2 \pi d}{\lambda} \sin \left[\theta_{m}+\phi\right]\right\}\left[u_{m} z_{m}^{*}+u_{m}^{*} z_{m}\right]+\frac{32 \pi^{2} \rho^{2}}{\lambda^{2}} \sin \left[\theta_{m}+\phi\right]^{2} \cos \left\{\frac{4 \pi d}{\lambda} \sin \left[\theta_{m}+\phi\right]\right\}$

The expectation of the second partial derivative is

$$
\begin{gathered}
E\left(\frac{\partial^{2} L}{\partial d^{2}}\right)=-\frac{1}{\sigma_{w}^{2}} \sum_{m=0}^{M-1} \frac{8 \pi^{2} \rho}{\lambda} \sin \left[\theta_{m}+\phi\right]^{2} \cos \left\{\frac{2 \pi d}{\lambda} \sin \left[\theta_{m}+\phi\right]\right\}\left\{u_{m} E\left[z_{m}^{*}\right]+u_{m}^{*} E\left[z_{m}\right]\right\} \\
+\frac{32 \pi^{2} \rho^{2}}{\lambda^{2}} \sin \left[\theta_{m}+\phi\right]^{2} \cos \left\{\frac{4 \pi d}{\lambda} \sin \left[\theta_{m}+\phi\right]\right\} .
\end{gathered}
$$

Based on (50), we have

$$
E\left[z_{m}\right]=2 \rho \cos \left\{\frac{2 \pi d}{\lambda} \sin \left[\theta_{m}+\phi\right]\right\} u_{m} \text {. }
$$

Thus (A.3) becomes

$$
E\left(\frac{\partial^{2} L}{\partial d^{2}}\right)=-\frac{32 \pi^{2} \rho^{2}}{\sigma_{w}^{2} \lambda^{2}} \sum_{m=0}^{M-1} \sin \left[\theta_{m}+\phi\right]^{2} \sin \left\{\frac{2 \pi d}{\lambda} \sin \left[\theta_{m}+\phi\right]\right\}^{2}
$$




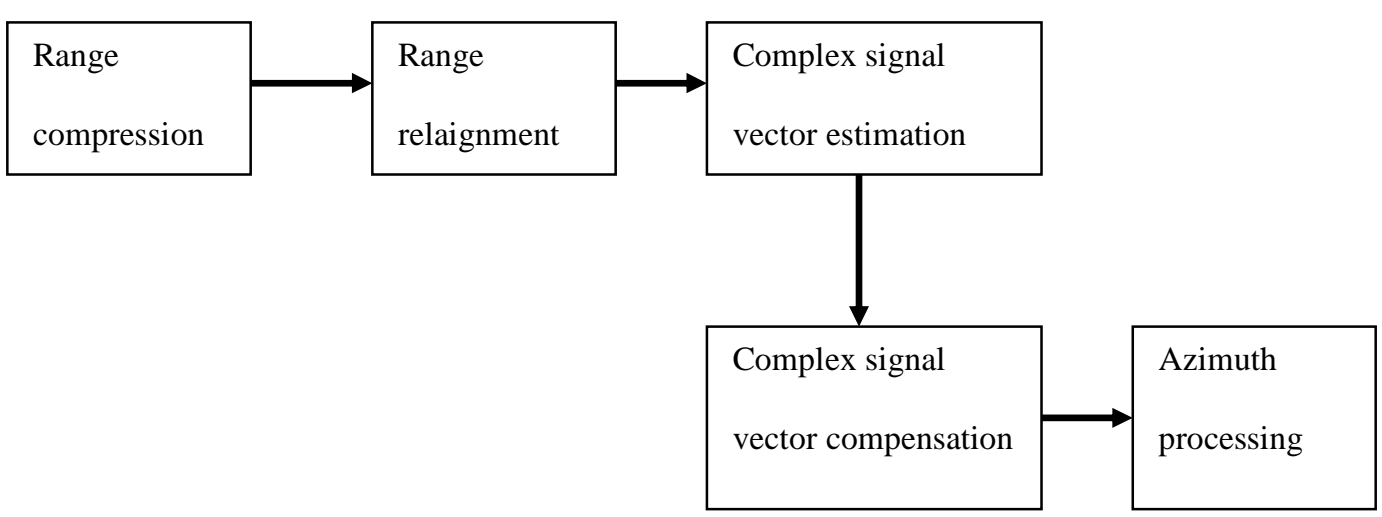

Fig. 1

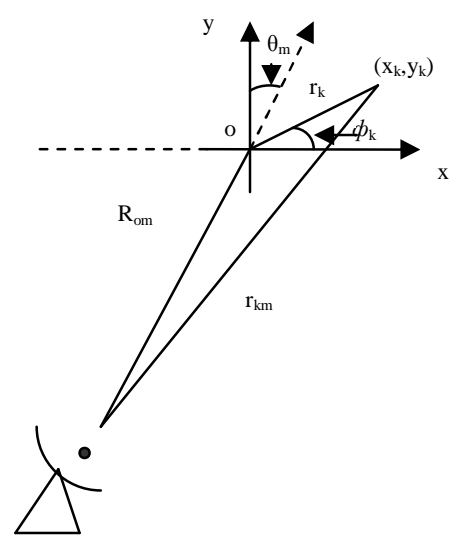

Fig. 2

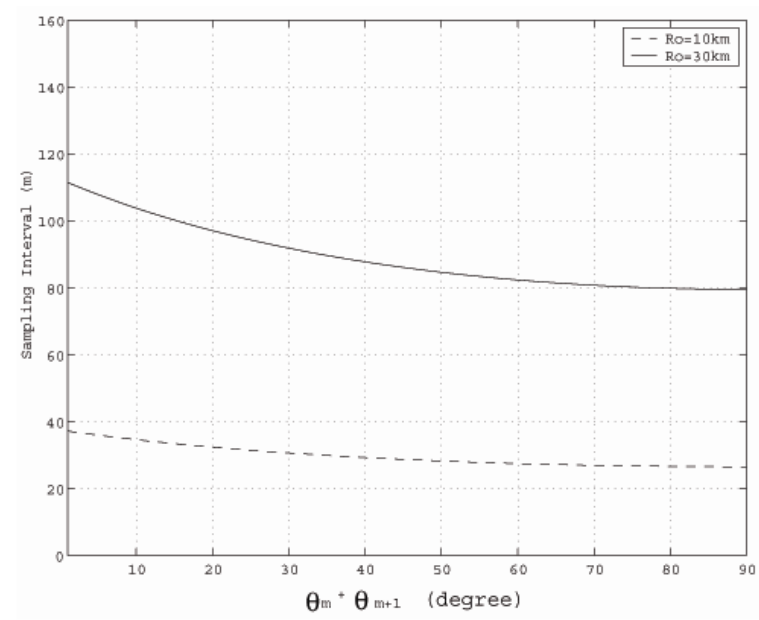

Fig. 3 


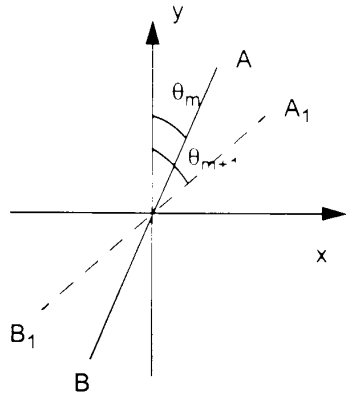

Fig. 4

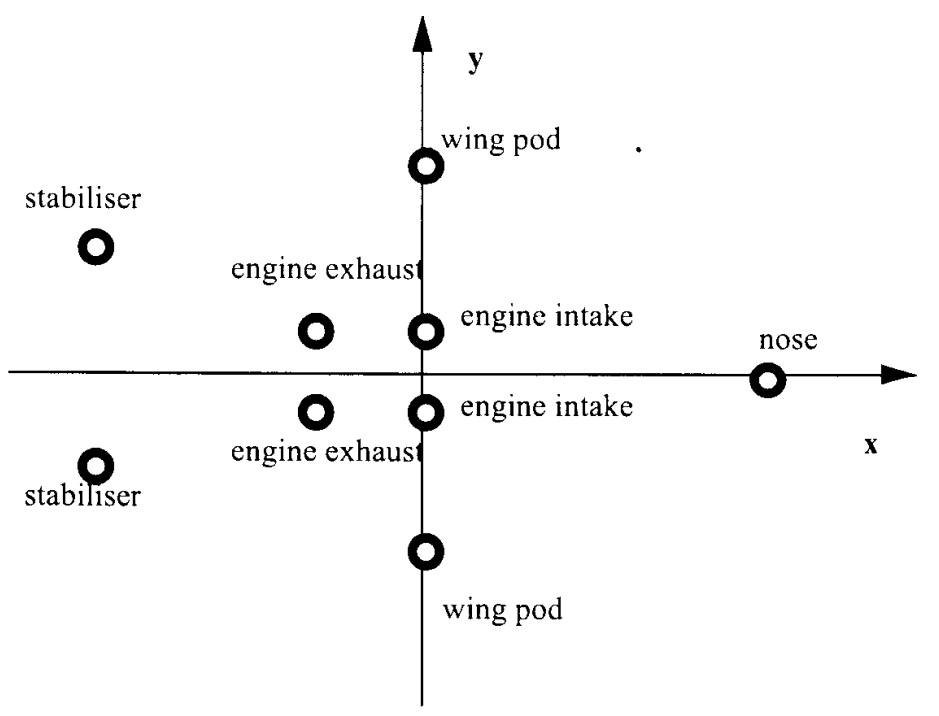

Fig. 5

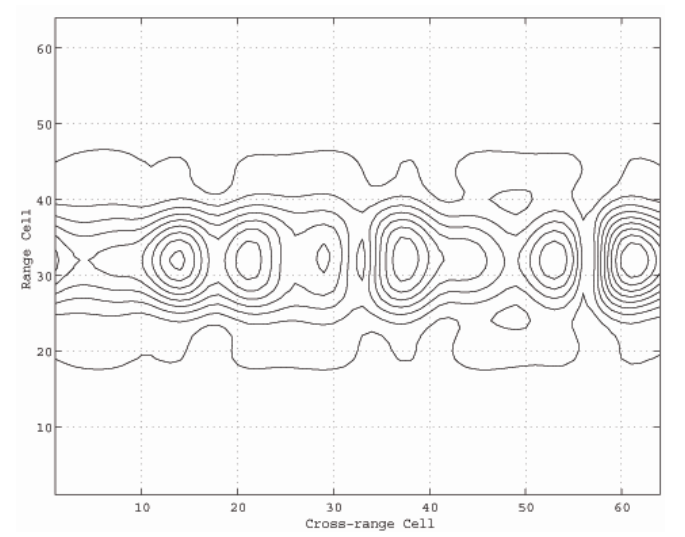

(a)

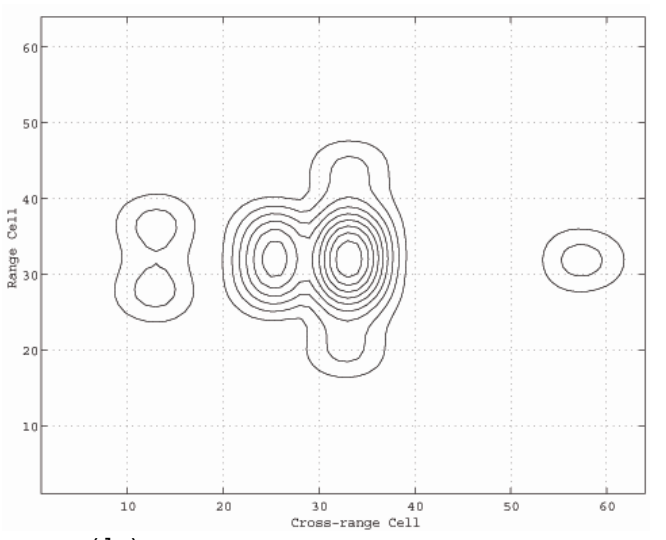

(b) 


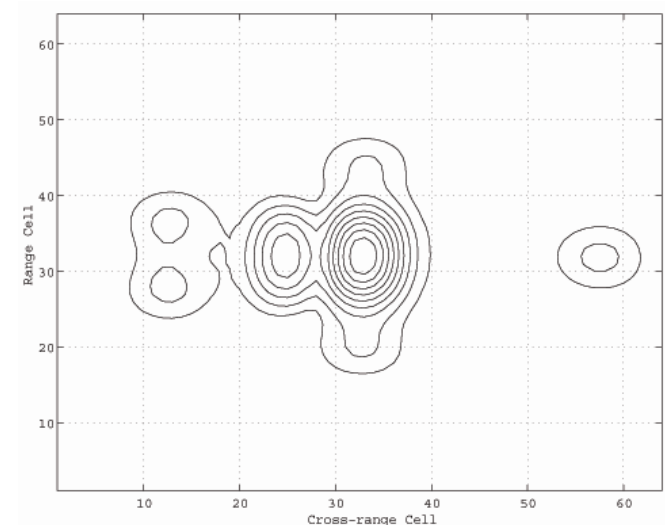

(c)

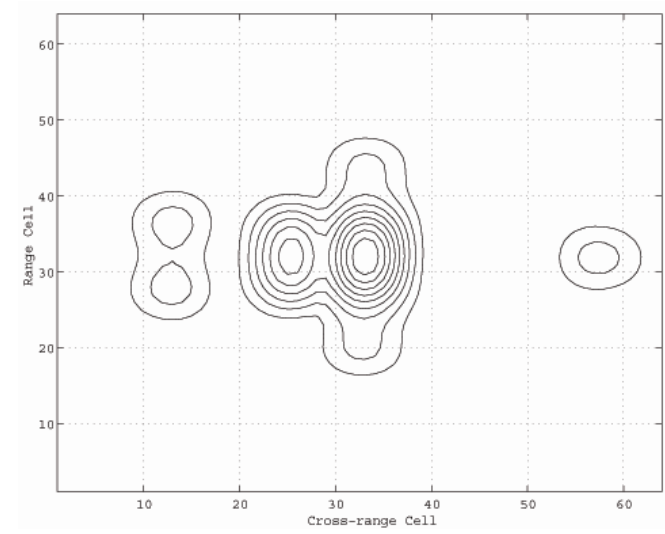

(e)

Fig. 6

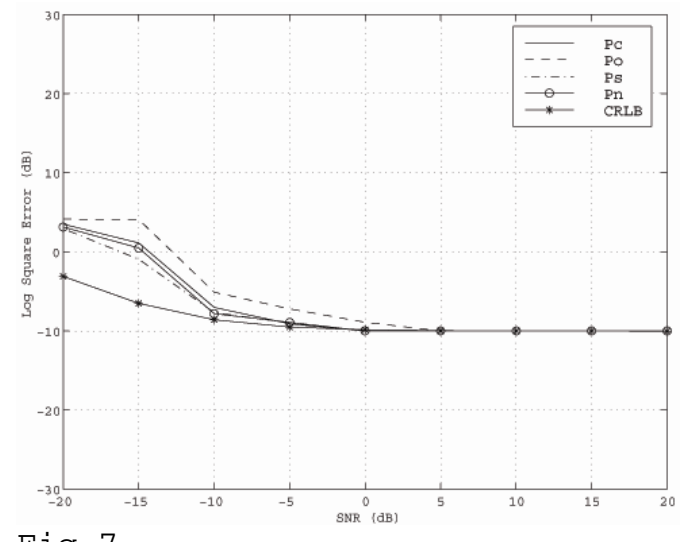

Fig. 7

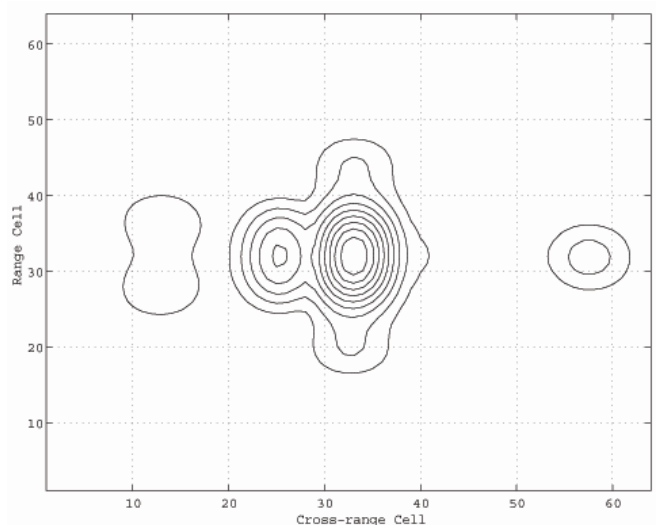

(d) 


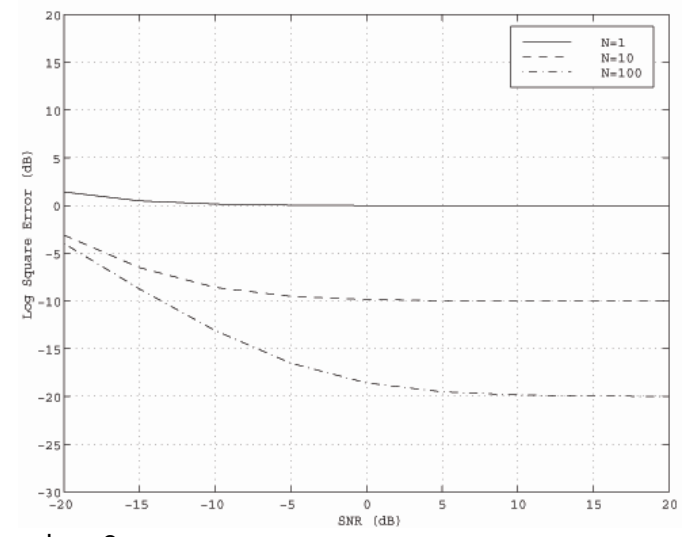

Fig. 8

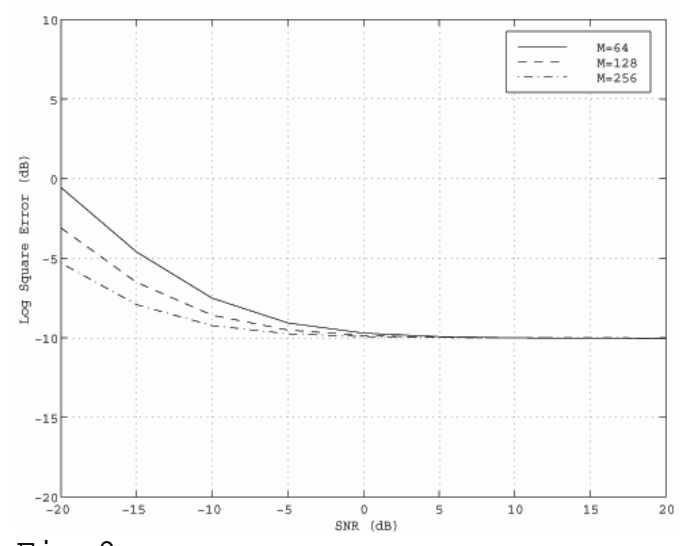

Fig. 9

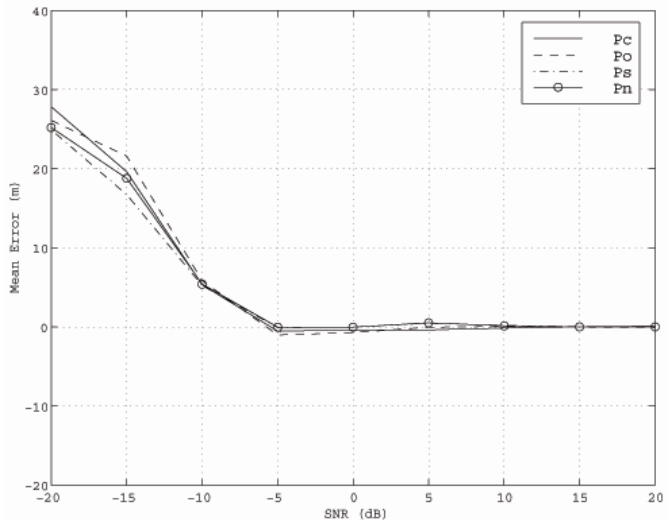

(a)

Fig. 10

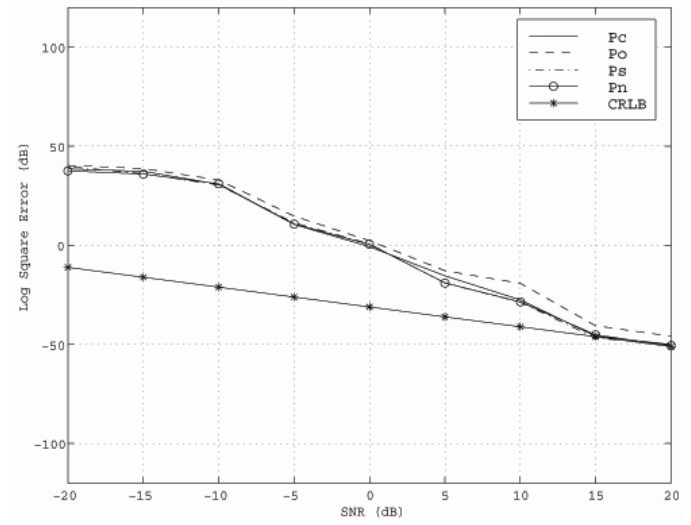

(b) 


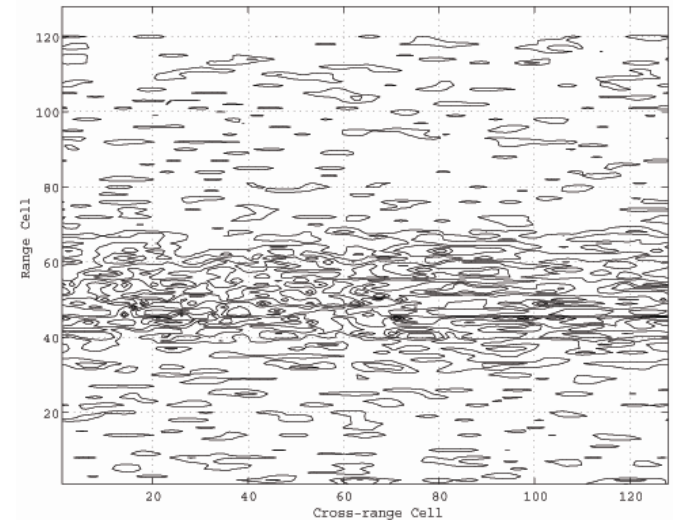

(a)

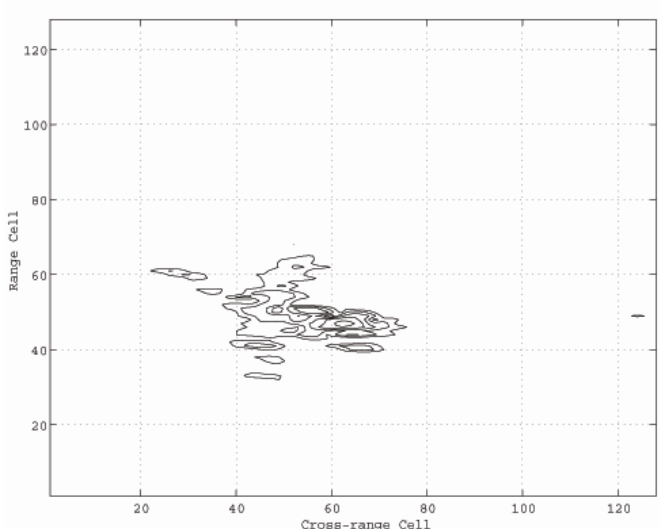

(c)

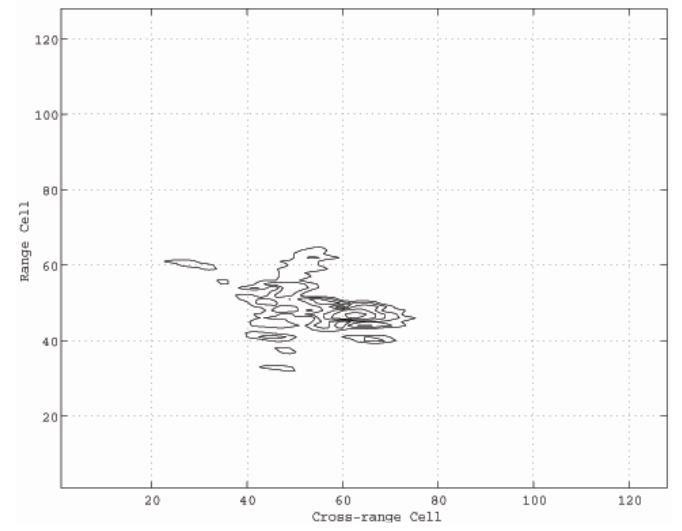

(e)

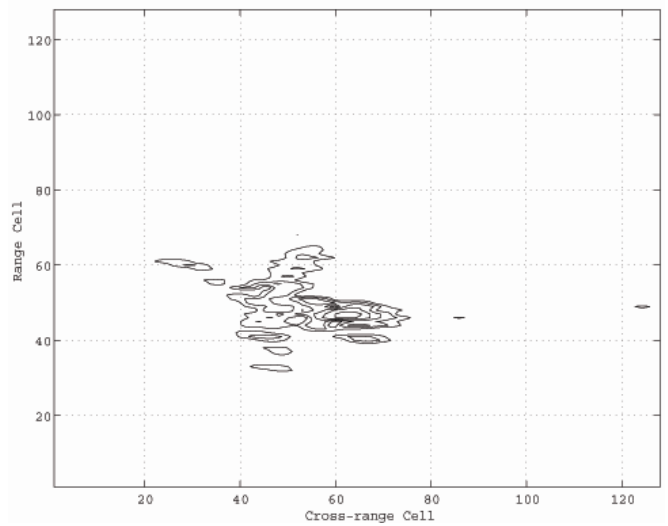

(b)

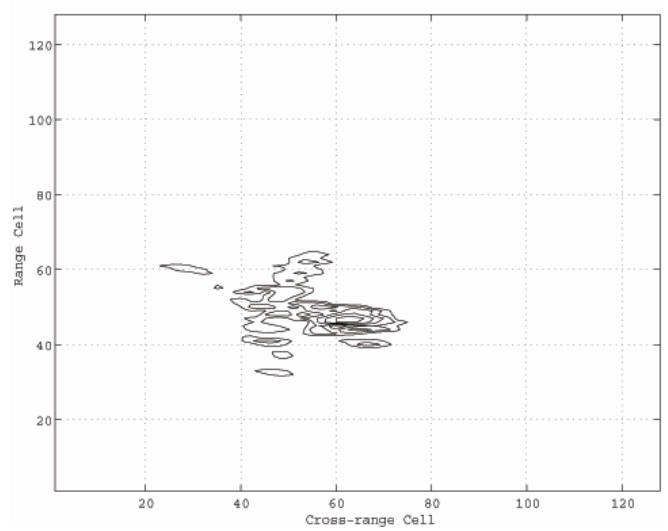

(d)

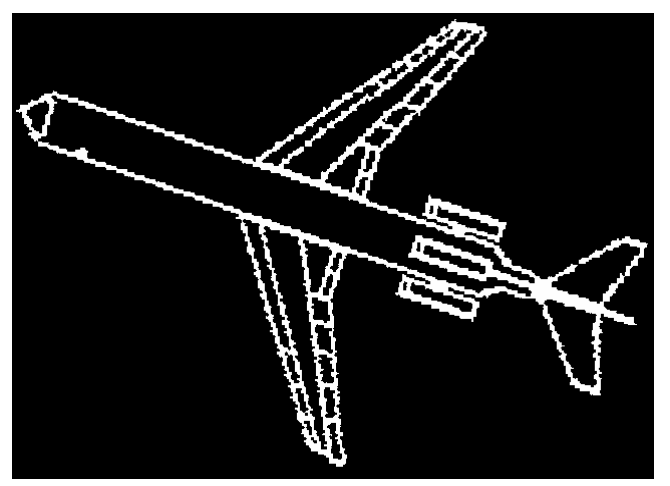

(f)

Fig.11 